\title{
Ehrenfeucht-Mostowski models in Abstract Elementary Classes
}

\author{
John T. Baldwin \\ Department of Mathematics, Statistics and Computer Science \\ University of Illinois at Chicago
}

We work in the context of an abstract elementary class (AEC) with the amalgamation and joint embedding properties and arbitrarily large models. We prove two results using Ehrenfeucht-Mostowski models: 1) Morley's omitting types theorem - for Galois types. 2) If an AEC (with amalgamation) is categorical in some uncountable power $\mu$ it is stable in (every) $\lambda<\mu$.

These results are lemmas towards Shelah's consideration [12] of the downward transfer of categoricity, which we discuss in Section 6. This paper expounds some of the main ideas of [12], filling in vague allusions to earlier work and trying to separate those results which depend only on the Ehrenfeucht-Mostowski method from those which require more sophisticated stability theoretic tools.

In [15], Shelah proclaims the aim of reconstructing model theory, 'with no use of even traces compactness'. We analyze here one aspect of this program. Keisler organizes [8] around four kinds of constructions: the Henkin method, EhrenfeuchtMostowski models, unions of chains, and ultraproducts. The later history of model theory reveals a plethora of tools arising in stability theory. Fundamental is a notion of dependence which arises from Morley's study of rank, and passes through various avatars of splitting, strong splitting, and dividing before being fully actualized in the first order setting as forking. We eschew this technique altogether in this paperto isolate its role.

The axioms of an AEC $(\boldsymbol{K}, \preceq \boldsymbol{K})$, were first set down in [17]. We repeat for convenience.

Definition 0.1. A class of L-structures, $(\boldsymbol{K}, \preceq \boldsymbol{K})$, is said to be an abstract elementary class: AEC if both $\boldsymbol{K}$ and the binary relation $\preceq \boldsymbol{K}$ are closed under isomorphism and satisfy the following conditions.

- A1. If $M \preceq \boldsymbol{K} N$ then $M \subseteq N$.

- A2. $\preceq \boldsymbol{K}$ is a partial order on $\boldsymbol{K}$.

- A3. If $\left\langle A_{i}: i<\delta\right\rangle$ is $\preceq \boldsymbol{K}^{\text {-increasing chain: }}$

(C)0000 (copyright holder) 
(1) $\bigcup_{i<\delta} A_{i} \in \boldsymbol{K}$

(2) for each $j<\delta, A_{j} \preceq \boldsymbol{K} \bigcup_{i<\delta} A_{i}$

(3) if each $A_{i} \preceq \boldsymbol{K} M \in \boldsymbol{K}$ then $\bigcup_{i<\delta} A_{i} \preceq \boldsymbol{K} M$.

- A4.[Coherence Axiom] If $A, B, C \in \boldsymbol{K}, A \preceq \boldsymbol{K} C, B \preceq \boldsymbol{K} C$ and $A \subseteq B$ then $A \preceq \boldsymbol{K} B$.

- A5. There is a Löwenheim-Skolem number $\kappa(\boldsymbol{K})$ such that if $A \subseteq B \in K$ there is a $A^{\prime} \in \boldsymbol{K}$ with $A \subseteq A^{\prime} \preceq \boldsymbol{K} B$ and $\left|A^{\prime}\right|<|A|+\kappa(\boldsymbol{K})$.

In English, we often write $B$ is a strong extension of $A$ for $A \preceq \boldsymbol{K} B$.

Sections 1, 2, 3 define most of the terminology and lay out the basic results. In Sections 4, we show categoricity implies stability and establish the existence of saturated models. Section 5 lifts Morley's omitting types theorem to the AEC setting. Finally in Section 6, we survey the additional steps needed to prove Shelah's downward categoricity theorem. I thank Greg Cherlin for some trenchant observations, Tapani Hyttinen for pointing out an error in an earlier draft, and Alex Usvyatsov for a careful reading.

\section{Assumptions}

We work with classes of structures in a fixed vocabulary, $\tau$. When results are uniform functions of such invariants as the cardinality of $\tau$ or $\operatorname{LS}(\boldsymbol{K})$ we may write them in terms of these numbers. We use variants on $\tau$ to denote vocabularies. In addition to this usage, Shelah uses $\tau$ as an operator: $\tau(\Phi)$ denotes the vocabulary of the set of sentences $\Phi$. We may write $\tau$-structure or $L$-structure.

Assumption 1.1. $\quad$ (1) $\boldsymbol{K}$ has arbitrarily large models.

(2) $\boldsymbol{K}$ satisfies the amalgamation property and the joint embedding property.

We say $\boldsymbol{K}$ has the amalgamation property if $M, N_{1}, N_{2} \in \boldsymbol{K}$ and there are strong embeddings of $M$ into $N_{1}$ and $N_{2}$ then there is an $N_{3}$ and strong embeddings of $N_{1}$ and $N_{2}$ into $N_{3}$ so that the composition maps agree on $M$. Joint embedding means that for any two members of $\boldsymbol{K}$ there is a third into which both can be strongly embedded. Crucially, we amalgamate only over members of $\boldsymbol{K}$; this distinguishes this context from the context of homogeneous structures. Amalgamation does not imply the existence of arbitrarily large models; the class of initial segments of $\aleph_{1}$ with end extension as strong extension is an AEC. An AEC with disjoint amalgamation (the images of $N_{1}$ and $N_{2}$ in $N_{3}$ intersect in the image of $M$ ) and at least two models can easily be seen to have arbitrarily large models.

We stress that amalgamation is a very strong assumption and we make full use of it. However, many of the results can be achieved under some weaker conditions with somewhat more effort; we allude to some of these. Much of the Shelah work involves two kinds of argument of a more local nature: failure of amalgamation in $\kappa$ implies many models in, say, $\kappa^{+}$(with various variants), and arguments which assume only amalgamation below (or in) a certain cardinality.

Notation 1.2. (1) Let $\mu(\lambda, \kappa)$ be the least cardinal $\mu$ such that if a first order theory $T$ with $|T|=\lambda$ has models of every cardinal less than $\mu$ which omit 
each of a set $\Gamma$ of types, with $|\Gamma|=\kappa$, then there are arbitrarily large models of $T$ which omit $\Gamma$.

(2) Write $\mu(\kappa)$ for $\mu(\kappa, \kappa)$.

(3) We say $\lambda$ is substantial for $\kappa$ if $\lambda \geq \mu(\kappa)$.

(4) For a similarity type $\tau, \mu(\tau)$ means $\mu(|\tau|)$.

Note that an old theorem of Morley [11], VII.5, [3] says $\mu(\kappa, \kappa) \leq \beth_{\left(2^{\kappa}\right)^{+}}$. For simplicity, we assume the Löwenheim number is at least $|\tau|$.

When $\operatorname{LS}(\boldsymbol{K})=|\tau(\boldsymbol{K})|=\kappa, \mu(\kappa)$ is sometimes called the Hanf number of $\boldsymbol{K}$. This is somewhat misleading because a single class cannot have a Hanf number - a Hanf number is a maximum for all similarity types of a given cardinality. It is in fact not the Hanf number of $\boldsymbol{K}$ but the Hanf number for all AEC with the same Löwenheim number. But as we'll see there is a still wider basis for this name; we will consider other classes of models (which are not AEC) and it is crucial that all of them have the property: for any model $M$ with $|M| \geq \mu(\tau)$, there are models in the class of all cardinalities that omit all types omitted in $M$.

There is some vestige of compactness here. Both the existence of arbitrarily large models and amalgamation are proved in first order logic using compactness. But they have completely semantic statements and you have to start somewhere.

\section{The presentation theorem and E-M models}

We call the next result: the presentation theorem. It allows us to replace the entirely semantic description of an abstract elementary class by a syntactic one. I find it extraordinary that the notion of an AEC which is designed to give a version of the Fraïsse construction and thus saturated models, also turns out to allow the use of the second great model theoretic technique of the 50's: Ehrenfeucht-Mostowski models.

THEOREM 2.1. If $K$ is an AEC with Lowenheim number $\operatorname{LS}(\boldsymbol{K})$ (in a vocabulary $\tau$ with $|\tau| \leq \operatorname{LS}(\boldsymbol{K})$ ), there is a vocabulary $\tau^{\prime}$ with cardinality $|\mathrm{LS}(\boldsymbol{K})|$, a first order $\tau^{\prime}$-theory $T^{\prime}$ and a set of $2^{\mathrm{LS}(\boldsymbol{K})}$ types $\Gamma$ such that:

$$
\boldsymbol{K}=\left\{M^{\prime} \mid \tau: M^{\prime} \models T^{\prime} \text { and } M^{\prime} \text { omits } \Gamma\right\} .
$$

Moreover, if $M^{\prime}$ is a $\tau^{\prime \prime}$-substructure of $N^{\prime}$ where $M^{\prime}, N^{\prime}$ satisfy $T^{\prime}$ and omit $\Gamma$ then $M^{\prime} \uparrow \tau \preceq K N^{\prime} \uparrow \tau$.

Proof. Let $\tau^{\prime}$ contain $n$-ary function symbols $F_{i}^{n}$ for $n<\omega$ and $i<\operatorname{LS}(\boldsymbol{K})$. We take as $T^{\prime}$ the theory which asserts only that its models are nonempty. For any $\tau^{\prime}$-structure $M^{\prime}$ and any $\boldsymbol{a} \in M$, let $M_{\boldsymbol{a}}^{\prime}$ denote the subset of $M^{\prime}$ enumerated as $\left\{F_{i}^{n}(\boldsymbol{a}): i<\operatorname{LS}(\boldsymbol{K})\right\}$ where $n=\lg (\boldsymbol{a})$; the only requirement on this enumeration is that the first $n$-elements are $\boldsymbol{a}$. The isomorphism type of ' $\boldsymbol{a}$ is determined by the quantifier free $\tau^{\prime}$-type of $\boldsymbol{a}$. Note that $M_{\boldsymbol{a}}^{\prime}$ may not be either a $\tau^{\prime}$ or even a $\tau$-structure. Let $\Gamma$ be the set of quantifier free $\tau^{\prime}$-types of finite tuples $\boldsymbol{a}$ such that $M_{\boldsymbol{a}}^{\prime}\left\lceil\tau \notin \boldsymbol{K}\right.$ or for some $\mathbf{b} \subset \boldsymbol{a}, M_{\mathbf{b}}^{\prime}\left\lceil\tau \npreceq \boldsymbol{K} M_{\boldsymbol{a}}^{\prime}\lceil\tau\right.$. 
We claim $T^{\prime}$ and $\Gamma$ suffice. That is, if $\boldsymbol{K}^{\prime}=\left\{M^{\prime} \uparrow \tau: M^{\prime} \models T^{\prime}\right.$ and $M^{\prime}$ omits $\left.\Gamma\right\}$ then $\boldsymbol{K}=\boldsymbol{K}^{\prime}$. Let the $\tau^{\prime}$-structure $M^{\prime}$ omit $\Gamma$; in particular, each $M_{\boldsymbol{a}}^{\prime}$ is a $\tau$ structure. Write $M^{\prime}$ as a direct limit of the finitely generated $\tau$-structures $M_{\boldsymbol{a}}^{\prime}$. (These may not be closed under the operations of $\tau^{\prime}$.) By the choice of $\Gamma$, each $M_{\boldsymbol{a}}^{\prime}\left\lceil\tau \in \boldsymbol{K}\right.$ and if $\boldsymbol{a} \subseteq \boldsymbol{a}^{\prime}, M_{\boldsymbol{a}}^{\prime}\left\lceil\tau \preceq \boldsymbol{K} M_{\boldsymbol{a}^{\prime}}^{\prime} \uparrow \tau\right.$, and so by the unions of chains axioms $M^{\prime} \mid \tau \in \boldsymbol{K}$. Conversely, if $M \in \boldsymbol{K}$ we define by induction on $|\boldsymbol{a}|$, structures $M_{\boldsymbol{a}}$ for each finite subset $\boldsymbol{a}$ of $M$. Let $M_{\emptyset}$ be any $\preceq \boldsymbol{K}^{\text {-substructure of }}$ $M$ with cardinality $\operatorname{LS}(\boldsymbol{K})$ and let the $\left\{F_{i}^{0}: i<\operatorname{LS}(\boldsymbol{K})\right\}$ be constants enumerating the universe of $M_{\emptyset}$. Given a sequence $\mathbf{b}$ of length $n+1$, choose $M_{\mathbf{b}} \preceq \boldsymbol{K} M$ with cardinality $\operatorname{LS}(\boldsymbol{K})$ containing all the $M_{\boldsymbol{a}}$ for $\boldsymbol{a} \subset \mathbf{b}$ of smaller cardinality. Let $\left\{F_{i}^{n+1}(\mathbf{b}): i<\operatorname{LS}(\boldsymbol{K})\right\}$ enumerate the universe of $M_{\mathbf{b}}$ (and give the function the same value on any ordering of the range of $\mathbf{b}$ ). Now each $M_{\boldsymbol{a}} \mid \tau \in \boldsymbol{K}$ and if $\mathbf{b} \subset \mathbf{c}$, $M_{\mathbf{b}} \preceq \boldsymbol{K} M_{\mathbf{c}}$ so $M^{\prime}$ omits $\Gamma$ as required.

The moreover holds for the partial $\tau^{\prime}$-structures $M_{\boldsymbol{a}}^{\prime}$ directly by the choice of $\Gamma$ and extends to arbitrary structures by the union of chain axioms on an AEC. In more detail, we have $M^{\prime}$ is a direct limit of finite structures $M_{\boldsymbol{a}}^{\prime}$ and $N^{\prime}$ is a $\preceq \boldsymbol{K}^{\text {-direct }}$ limit of $N_{\boldsymbol{a}}^{\prime}$ where $M_{\boldsymbol{a}}^{\prime}=N_{\boldsymbol{a}}^{\prime}$ for $\boldsymbol{a} \in M$ because $M^{\prime} \uparrow \tau$ is a $\tau$-substructure of $N^{\prime} \uparrow \tau$. Each $M_{\boldsymbol{a}}^{\prime}\left\lceil\tau \preceq \boldsymbol{K} N^{\prime} \uparrow \tau\right.$ so the direct limit $M^{\prime} \uparrow \tau$ is a strong submodel of $N^{\prime} \uparrow \tau . \quad \square_{2.1}$

We have represented $\boldsymbol{K}$ as a $P C \Gamma$ class in the following sense.

Definition 2.2. A PC $(T, \Gamma)$ class is the class of reducts to $\tau \subset \tau^{\prime}$ of models of a first order theory $\tau^{\prime}$-theory which omit all types from the specified collection $\Gamma$ of types in finitely many variables over the empty set.

We write $P C \Gamma$ to denote such a class without specifying either $T$ or $\Gamma$. And we write $\boldsymbol{K}$ is $P C(\lambda, \mu)$ if $\boldsymbol{K}$ can be presented as $P C(T, \Gamma)$ with $|T| \leq \lambda$ and $|\Gamma| \leq \mu$. In the simplest case, we say $\boldsymbol{K}$ is $\lambda$-presented if $\boldsymbol{K}$ is $P C(\lambda, \lambda)$.

In this language we have shown any $\mathrm{AEC} \boldsymbol{K}$ is $2^{\mathrm{LS}(\mathrm{K})}$-presented.

REMARK 2.3. (1) There is no use of amalgamation in this theorem.

(2) The only penalty for increasing the size of the language or the Löwenheim number is that the size of $\tau^{\prime}$ and the nunber of types omitted increases as well. This will meant that for the use of EM models below, the $\theta$ must be chosen larger.

(3) We can observe with Shelah $[\mathbf{1 7}]$ that the class of pairs $(M, N)$ with $M \preceq K N$ also forms a $P C\left(\operatorname{LS}(\boldsymbol{K}), 2^{\mathrm{LS}(\boldsymbol{K})}\right)$. This observation is important for some applications but will not be used here; see Theorem 2.7 and its applications. The moreover clause appears in Grossberg's account: [5] and in Makowsky's [9].

We immediately conclude the required computation of Hanf numbers for abstract elementary classes; we will use in a significant way the fact that this is, in fact, the Hanf number for $P C \Gamma$ classes where $|\Gamma| \leq 2^{|\tau|}$.

COROllary 2.4. Let $\boldsymbol{K}$ be an AEC with similarity type $\tau$. If $\boldsymbol{K}$ has a model with cardinality at least $\mu(\tau)$ then $\boldsymbol{K}$ has arbitrarily large models. 
Notation 2.5. (1) For any linearly ordered set $X \subseteq M$ where $M$ is a $\tau$ structure we write $\mathbf{D}_{\tau}(X)$ (diagram) for the set of $\tau$-types of finite sequences (in the given order) from $X$. We will omit $\tau$ if it is clear from context.

(2) Such a diagram of an order indiscernible set, $\mathbf{D}_{\tau}(X)=\Phi$, is called 'proper for linear orders'.

(3) If $X$ is a sequence of $\tau$-indiscernibles with diagram $\Phi=\mathbf{D}_{\tau}(X)$ and any $\tau$ model of $\Phi$ has built in Skolem functions, then for any linear ordering I, $E M(I, \Phi)$ denotes the $\tau$-hull of a sequence of order indiscernibles realizing $\Phi$.

(4) If $\tau_{0} \subset \tau$, the reduct of $E M(I, \Phi)$ to $\tau_{0}$ is denoted $E M_{\tau_{0}}(I, \Phi)$.

'Morley's method' (Section 7.2 of [4]) is a fundamental technique in first order model theory. It is essential for the foundations of simplicity theory and for the construction of indiscernibles in infinitary logic. We quote the first order version here; in Lemma 5.1, we prove the analog for abstract elementary classes.

LEMma 2.6. If $(X,<)$ is a sufficiently long linearly ordered subset of a $\tau$-structure $M$, for any $\tau^{\prime}$ extending $\tau$ (the length needed for $X$ depends on $\left.\left|\tau^{\prime}\right|\right)$ with $<$ in $\tau^{\prime}$ there is a countable set $Y$ of $\tau^{\prime}$-indiscernibles (and hence one of arbitrary order type) such that $\mathbf{D}_{\tau}(Y) \subseteq \mathbf{D}_{\tau}(X)$. This implies that the only (first order) $\tau$-types realized in $\operatorname{EM}\left(X, \mathbf{D}_{\tau^{\prime}}(Y)\right)$ were realized in $M$.

Further, we find Skolem models over indiscernibles in an AEC.

TheOREM 2.7. If $\boldsymbol{K}$ is an abstract elementary class in the vocabulary $\tau$, which is presented as a $P C \Gamma$ class witnessed by $\tau^{\prime}, T^{\prime}, \Gamma$ that has arbitrarily large models, there is a $\tau^{\prime}$-diagram $\Phi$ such that for every linear order $(I,<)$ there is a $\tau^{\prime}$-structure $M=E M(I, \Phi)$ such that:

(1) $M \models T^{\prime}$.

(2) The $\tau^{\prime}$-structure $M=\operatorname{EM}(I, \Phi)$ is the Skolem hull of $I$.

(3) $I$ is a set of $\tau^{\prime}$-indiscernibles in $M$.

(4) $M \uparrow \tau$ is in $\boldsymbol{K}$.

(5) If $I^{\prime} \subset I$ then $\operatorname{EM}_{\tau}\left(I^{\prime}, \Phi\right) \preceq \boldsymbol{K} E M_{\tau}(I, \Phi)$.

Proof. The first four clauses are a direct application of Lemma 2.6, Morley's theorem on omitting types. See also problem 7.2.5 of Chang-Keisler [4] or [3]. It is automatic that $\operatorname{EM}\left(I^{\prime}, \Phi\right)$ is an $L^{\prime}$ substructure of $\operatorname{EM}(I, \Phi)$. The moreover clause of Theorem 2.1 allows us to extend this to $E M_{\tau}\left(I^{\prime}, \Phi\right) \preceq K E M_{\tau}(I, \Phi) . \quad \square_{2.7}$

Note that we have simplified our presentation of many members of $\boldsymbol{K}$. Inside the class $\boldsymbol{K}$, which is the set of reducts of models which omit $\Gamma$, sits a class $\boldsymbol{K}^{\prime}$, which is the class of reducts of Skolem hulls of order indiscernibles. In general, $\boldsymbol{K}^{\prime}$ is a proper subclass of $\boldsymbol{K}$. It may not be an AEC because we don't know closure under unions of chains. In [14], under strong hypotheses this closure is proved.

REMARK 2.8. Silver (Chapter 18 of [8]) gives a simple example of a psuedoelementary class where the categoricity spectrum and its complement are both cofinal in 
the class of cardinals. The example is the class of models $(M, X)$ where $2^{|X|} \geq|M|$. This class is not an AEC because it is not closed under unions of chains.

The arguments below depend on classes being both AEC and $P C \Gamma$.

\section{Galois types and saturation}

In this section we take advantage of joint embedding and amalgamation to find a monster model. We then define types in terms of orbits of stabilizers of submodels. This allows an identification of 'model-homogeneous' with 'saturated'. That is, we give an abstract account of Morley-Vaught [10].

Definition 3.1. $M$ is $\mu$-model homogenous if for every $N \preceq \boldsymbol{K} M$ and every $N^{\prime} \in \boldsymbol{K}$ with $\left|N^{\prime}\right|<\mu$ and $N \preceq \boldsymbol{K} N^{\prime}$ there is a $\boldsymbol{K}$-embedding of $N^{\prime}$ into $M$ over $N$.

To emphasize, this differs from the homogenous context because the $N$ must be in $\boldsymbol{K}$. It is easy to show:

LEMmA 3.2. If $M_{1}$ and $M_{2}$ are $\mu$-model homogenous of cardinality $\mu>\operatorname{LS}(\boldsymbol{K})$ then $M_{1} \approx M_{2}$.

Proof. If $M_{1}$ and $M_{2}$ have a common submodel $N$ of cardinality $<\mu$, this is an easy back and forth. Now suppose $N_{1},\left(N_{2}\right)$ is a small model of $M_{1},\left(M_{2}\right)$ respectively. By the joint embedding property there is a small common extension $N$ of $N_{1}, N_{2}$ and by model homogeneity $N$ is embedded in both $M_{1}$ and $M_{2} . \quad \square_{3.2}$

Note that in the absence of joint embedding, to get uniqueness we would (as in [17]) have to add to the definition of ' $M$ is model homogeneous' that all models of cardinality $<\mu$ are embedded in $M$.

THEOREM 3.3. If $\boldsymbol{K}$ has the amalgamation property and $\mu *<\mu *=\mu *$ and $\mu * \geq$ $2^{\mathrm{LS}(\boldsymbol{K})}$ then there is a model $\mathbb{M}$ of cardinality $\mu *$ which is $\mu *$-model homogeneous.

We call the model constructed in Theorem 3.3, the monster model. From now on all, structures considered are substructures of $\mathbb{M}$ with cardinality $<\mu *$. The standard arguments for the use of a monster model in first order model theory $([\mathbf{7}, \mathbf{2}]$ apply here.

Definition 3.4. Let $M \in \boldsymbol{K}, M \preceq K \mathbb{M}$ and $a \in \mathbb{M}$. The Galois type of a over $M$ is the orbit of a under the automorphisms of $\mathbb{M}$ which fix $M$.

We freely use the phrase, 'Galois type of $a$ over $M$ '. Note that a priori this notion depends on the embedding of $M a$ into an $N \in \boldsymbol{K}$ and the embedding of $N$ into $\mathbb{M}$. Since we have assumed amalgamation, our usage is justified as long as the base is an $M \in \boldsymbol{K}$. In more general situations, the Galois type is an equivalence class of an equivalence relation on triples $(M, a, N)$. This is an equivalence relation on the class of $M$ that are amalgamation bases for extensions in the same cardinality. (See $[\mathbf{1 8}, \mathbf{1 9}]$.) Since we have amalgamation and have fixed $\mathbb{M}$, we don't need the extra notation. The following definition and exercise show the connection of the 
situation as described here with the more complicated description elsewhere. They are needed only to link with the literature.

Definition 3.5. For $M \preceq \boldsymbol{K} N_{1} \in \boldsymbol{K}, M \preceq \boldsymbol{K} N_{1} \in \boldsymbol{K}$ and $a \in N_{1}-M$, $b \in N_{2}-M$, write $\left(M, a, N_{1}\right) \sim\left(M, b, N_{2}\right)$ if there exist strong embeddings $f_{1}, f_{2}$ of $N_{1}, N_{2}$ into some $N^{*}$ which agree on $M$ and with $f_{1}(a)=f_{2}(b)$.

EXERCISE 3.6. If $\boldsymbol{K}$ has amalgamation, $\sim$ is an equivalence relation.

EXERCISE 3.7. Suppose $\boldsymbol{K}$ has amalgamation and joint embedding. Show $\left(M, a, N_{1}\right) \sim\left(M, b, N_{2}\right)$ if and only if there are embeddings $g_{1}$ and $g_{2}$ of $N_{1}, N_{2}$ into $\mathbb{M}$ that agree on $M$ and such that $g_{1}(a)$ and $g_{2}(b)$ have the same Galois type over $g_{1}(M)$.

Definition 3.8. The set of Galois types over $M$ is denoted ga - S(M).

We say a Galois type $p$ over $M$ is realized in $N$ with $M \preceq \boldsymbol{K} N \preceq \boldsymbol{K} \mathbb{M}$ if $p \cap N \neq \emptyset$.

Definition 3.9. The model $M$ is $\mu$-Galois saturated if for every $N \preceq \boldsymbol{K} M$ with $|N|<\mu$ and every Galois type $p$ over $N, p$ is realized in $M$.

Again, a priori this notion depend on the embedding of $M$ into $\mathbb{M}$; but with amalgamation it is well-defined.

ThEOREM 3.10. For $\lambda>\operatorname{LS}(\boldsymbol{K})$, The model $M$ is $\lambda$-Galois saturated if and only if it is $\lambda$-model homogeneous.

Proof. It is obvious that $\lambda$-model homogeneous implies $\lambda$-Galois saturated. Let $M \preceq \boldsymbol{K} \mathbb{M}$ be $\lambda$-saturated. We want to show $M$ is $\lambda$-model homogeneous. So fix $M_{0} \preceq \boldsymbol{K} M$ and $N$ with $M_{0} \preceq \boldsymbol{K} N \preceq \boldsymbol{K} \mathbb{M}$. Say, $|N|=\mu<\lambda$. We must construct an embedding of $N$ into $M$. Enumerate $N-M$ as $\left\langle a_{i}: i<\mu\right\rangle$. We will define $f_{i}$ for $i<\mu$ an increasing continuous sequence of maps with domain $N_{i}$ and range $M_{i}$ so that $M_{0} \preceq \boldsymbol{K} N_{i} \preceq \boldsymbol{K} \mathbb{M}, M_{0} \preceq \boldsymbol{K} M_{i} \preceq \boldsymbol{K} M$ and $a_{i} \in N_{i+1}$. The restriction of $\bigcup_{i<\mu} f_{i}$ to $N$ is required embedding. Let $N_{0}=M_{0}$ and $f_{0}$ the identity. Suppose $f_{i}$ has been defined. Choose the least $j$ such that $a_{j} \in N-N_{i}$. By the model homogeneity of $\mathbb{M}, f_{i}$ extends to an automorphism $\hat{f}_{i}$ of $\mathbb{M}$. Using the saturation, let $b_{j} \in M$ realize the Galois type of $\hat{f}_{i}\left(a_{j}\right)$ over $M_{i}$. So there is an $\alpha \in$ aut $\mathbb{M}$ which fixes $M_{i}$ and takes $b_{j}$ to $\hat{f}_{i}\left(a_{j}\right)$. Choose $M_{i+1} \preceq \boldsymbol{K} M$ with cardinality $\mu$ and containing $M_{i} b_{j}$. Now $\hat{f}_{i}^{-1} \circ \alpha$ maps $M_{i}$ to $N_{i}$ and $b_{j}$ to $a_{j}$. Let $N_{i+1}=\hat{f}_{i}^{-1} \circ \alpha\left(M_{i+1}\right)$ and define $f_{i+1}$ as the restriction of $\alpha^{-1} \circ \hat{f}_{i}$ to $N_{i+1}$. Then $f_{i+1}$ is as required. $\quad \square_{3.10}$

The last argument makes full use of the amalgamation property. We discuss some generalizations in the last paragraph of this article. In the remainder of this section we discuss some important ways in which Galois types behave differently from 'syntactic types'.

Note that if $M \preceq \boldsymbol{K} N \preceq \boldsymbol{K} \mathbb{M}$, then $p \in$ ga $-\mathrm{S}(\mathrm{N})$ extends $p^{\prime} \in$ ga $-\mathrm{S}(\mathrm{N})$ if for some (any) $a$ realizing $p$ and some (any) $b$ realizing $p^{\prime}$ there is an automorphism $\alpha$ fixing $M$ and taking $a$ to $b$. 
Lemma 3.11. If $M=\bigcup_{i<\omega} M_{i}$ is an increasing chain of members of $\boldsymbol{K}$ and $\left\{p_{i}\right.$ : $i<\omega\}$ satisfies $p_{i+1}\left\lceil M_{i}=p_{i}\right.$, there is a $p_{\omega} \in$ ga $-\mathrm{S}(\mathrm{M})$ with $p_{\omega}\left\lceil M_{i}=p_{i}\right.$ for each $i$.

Proof. Let $a_{i}$ realize $p_{i}$. By hypothesis, for each $i<\omega$, there exists $f_{i}$ which fixes $M_{i-1}$ and maps $a_{i}$ to $a_{i-1}$. Let $g_{i}$ be the composition $f_{0} \circ f_{1} \circ \ldots f_{i}$. Then $g_{i}$ maps $a_{i}$ to $a_{0}$, fixes $M_{0}$ and $g_{i} \uparrow M_{i-1}=g_{i-1} \uparrow M_{i-1}$. Let $M_{i}^{\prime}$ denote $g_{i}\left(M_{i}\right)$ and $M^{\prime}$ their union. Then $\bigcup_{i<\omega} g_{i}$ is an isomorphism between $M$ and $M^{\prime}$. So by model-homogeneity there exists an automorphism $h$ of $\mathbb{M}$ with $h\left\lceil M_{i}=g_{i}\left\lceil M_{i}\right.\right.$ for each $i$. Let $a_{\omega}=h^{-1}\left(a_{0}\right)$. Now $g_{i}^{-1} \circ h$ fixes $M_{i}$ and maps $a_{\omega}$ to $a_{i}$ for each $i$. This completes the proof. $\quad \square_{3.11}$

Now suppose we wanted to prove Lemma 3.11 for chains of length $\delta>\omega$. The difficulty can be seen at stage $\omega$. In addition to the assumptions of Lemma 3.11, we are given $\left\{a_{i}: i \leq \omega\right\}$ and $f_{\omega, i}$ which fixes $M_{i}$ and maps $a_{\omega}$ to $a_{i}$. We can construct $g_{i}$ as in the original proof. The difficulty is to find $g_{\omega}$ which extends all the $g_{i}$ and maps $a_{\omega}$ to $a_{0}$. In the argument for Lemma 3.11, we found a map $h$ and an element (which we will now call $a_{\omega}^{\prime}$ such that $h$ takes $a_{\omega}^{\prime}$ to $a_{0}$ while $h$ extends all the $g_{i}$. We would be done if $a_{\omega}$ and $a_{\omega}^{\prime}$ realized the same galois type over $M=M_{\omega}$. In fact, $a_{\omega}$ and $a_{\omega}^{\prime}$ realized the same Galois type over each $M_{i}$. So the following locality condition (for chains of length $\omega$ ) would suffice for this special case. Moreover, by a further induction locality would give Lemma 3.11 for chains of arbitrary length. Locality does not hold for all AEC with amalgamation; it would be interesting to find a concrete example. Locality is defined in Definition 24 of [15].

Definition 3.12. $\boldsymbol{K}$ has local Galois types if for every $M=\bigcup_{i<\kappa} M_{i}$ in a continuous increasing chain of members of $\boldsymbol{K}$ and for any $p, q \in \operatorname{ga}-\mathrm{S}(\mathrm{M})$ : if $p \nmid M_{i}=q \uparrow M_{i}$ for every $i$ then $p=q$.

We have sketched the proof of:

Lemma 3.13. Suppose $\boldsymbol{K}$ has local Galois types. If $M=\bigcup_{i<\kappa} M_{i}$ in an increasing chain of members of $\boldsymbol{K}$ and $\left\{p_{i}: i<\kappa\right\}$ satisfies $p_{i+1} \uparrow M_{i}=p_{i}$, there is a $p_{\kappa} \in \mathrm{ga}-\mathrm{S}(\mathrm{M})$ with $p_{\kappa} \uparrow M_{i}=p_{i}$ for each $i$.

Locality provides a key distinction between the general AEC case and homogenous structures. In homogeneous structures, types are syntactic objects and locality is trivial. Thus, as pointed out by Shelah, Hyttinen, and Buechler-Lessmann, Lemma 3.13 applies in the homogeneous context.

\section{Getting stability}

In this section we show that a countable $\lambda$-categorical AEC is $\mu$-stable for $\mu$ above the Löwenheim number and below $\lambda$. The key idea is that for a linear order $I$ and model $\operatorname{EM}(I, \Phi)$, automorphisms of I induce automorphisms of $E M(I, \Phi)$. And, automorphisms of $E M(I, \Phi)$ preserve types in any reasonable logic; in particular, automorphisms of $\operatorname{EM}(I, \Phi)$ preserve Galois types. Note that a model $N$ is (defined to be) stable if few types are realized in $N$. So if $N$ is a brimful model (Definition 4.2) then the model $N$ is $\sigma$-stable for every $\sigma<|N|$. 
Since we deal with reducts and will consider several structures with the same universe; it is crucial to keep the vocabulary of the structure in mind. The AEC under consideration has vocabulary $\tau$; it is presented as reducts of models of theory $T^{\prime}$ (which omit certain types) in a vocabulary $\tau^{\prime}$. In addition, we have the class of linear orderings $(L O)$ in the background.

We really have three AEC's: $(L O, \subset), \boldsymbol{K}^{\prime}$ which is $\operatorname{Mod}\left(T^{\prime}\right)$ with submodel as $\tau^{\prime}$ closed subset, and $(\boldsymbol{K}, \preceq \boldsymbol{K})$. We are describing the properties of the EM-functor between $(L O, \subset)$ and $\boldsymbol{K}^{\prime}$ or $\boldsymbol{K} . \boldsymbol{K}^{\prime}$ is only a tool that we are singling out to see the steps in the argument. The following definitions hold for any of the three classes and I write $\leq$ for the notion of substructure. In this section of the paper I am careful to use $\leq$ when discussing all three cases versus $\preceq \boldsymbol{K}$ for the AEC.

Definition 4.1. $M_{2}$ is $\sigma$-universal over $M_{1}$ in $N$ if $M_{1} \leq M_{2} \leq N$ and whenever $M_{1} \leq M_{2}^{\prime} \leq N$, with $\left|M_{1}\right| \leq\left|M_{2}^{\prime}\right| \leq \sigma$, there is $a \leq$-embedding fixing $M_{1}$ and taking $M_{2}^{\prime}$ into $M_{2}$.

I introduce one term for shorthand. It is related to Shelah's notion of brimmed in [13] but here the brimful model is bigger than the models it is universal over while brimmed models may have the same cardinality.

DEFinition 4.2. $M$ is brimful if for every $\sigma<|M|$, and every $M_{1} \leq M$ with $\left|M_{1}\right|=\sigma$, there is an $M_{2} \leq M$ with cardinality $\sigma$ that is $\sigma$-universal over $M_{1}$ in $M$.

The next notion just makes it easier to write the proof of the following Lemma.

Notation 4.3. Let $I \subset J$ be linear orders. We say a and $b$ in $J$ realize the same cut over $I$ and write $a \sim_{I} b$ if for every $i \in I, a<i$ if and only if $b<i$.

Claim 4.4 (Lemma 3.7 of [16]). The linear order $I=\lambda^{<\omega}$ is brimful.

Proof. Let $J \subset I$ have cardinality $\theta<\lambda$. Without loss of generality we can assume $J=A^{<\omega}$ for some $A \subset \lambda$. Note that $\sigma \sim{ }_{J} \tau$ if and only if for the least $n$ such that $\sigma \uparrow n \neq \tau \uparrow n$, neither is in $J$ and $\sigma(n) \sim_{A} \tau(n)$. Thus there are only $\theta$ cuts over $J$ realized in $I$. For each cut $C_{\alpha}, \alpha<\theta$, we choose a representative $\sigma_{\alpha} \in I-J$ of length $n$ such that $\sigma_{\alpha}\left\lceil n-1 \in J\right.$, so a cut in $J$ is isomorphic to $\left\{\sigma_{\alpha} \widehat{\tau}: \tau \in \lambda^{<\omega}, \alpha<\theta\right\}$. We can assume any $J^{*}$ extending $J$ is $J^{*}=B^{<\omega}$ for some $B \subset \lambda$, say with $\operatorname{otp}(B)=\gamma$. Thus, the intersection of $J^{*}$ with a cut in $J$ is isomorphic to a subset of $\gamma^{<\omega}$. We finish by noting for any ordinal $|\gamma|=\theta, \gamma^{<\omega}$ can be embedded in $\theta^{<\omega}$. Thus, the required $\theta$-universal set over $J$ is $J \cup\left\{\sigma_{\alpha} \widehat{\tau}: \tau \in \theta^{<\omega}, \alpha<\theta\right\}$.

Qing Zhang has provided the following elegant argument for the last claim. First show by induction on $\gamma$ there is a map $g$ embedding $\gamma$ in $\theta^{<\omega}$. (E.g. if $\gamma=$ $\lim _{i<\theta} \gamma_{i}$, and $g_{i}$ maps $\gamma_{i}$ into $\theta<\omega$, let for $\beta<\gamma, g(\beta)=\widehat{\imath} g_{i}(\beta)$ where $\gamma_{i} \leq$ $\beta<\gamma_{i+}$.) Then let $h$ map $\gamma^{<\omega}$ into $\theta^{<\omega}$ by, for $\sigma \in \gamma^{<\omega}$ of length $n$, setting $h(\sigma)=\langle g(\sigma(0)), \ldots, g(\sigma(n-1))\rangle . \quad \square_{4.4}$

The argument for Claim 4.4 yields:

Corollary 4.5. Suppose $\mu<\lambda$ are cardinals. Then for any $X \subset \mu^{<\omega}$ and any $Y$ with $X \subseteq Y \subset \lambda^{<\omega}$ and $|X|=|Y|<\mu$, there is an order embedding of $Y$ into $\mu^{<\omega}$ over $X$. 
Since every $\tau^{\prime}$-substructure $N$ of $\operatorname{EM}(I, \Phi)$ is contained in a substructure $\operatorname{EM}\left(I_{0}, \Phi\right)$ for some subset $I_{0}$ of $I$ with $\left|I_{0}\right|=|N|$, we have immediately:

Claim 4.6. If I is brimful as a linear order, $E M(I, \Phi)$ is brimful as an $\tau^{\prime}$-structure.

Now using amalgamation and categoricity, we move to the AEC $\boldsymbol{K}$. There are some subtle uses here of the 'coherence axiom': $M \subseteq N \preceq \boldsymbol{K} N_{1}$ and $M \preceq \boldsymbol{K} N_{1}$ implies $M \preceq \boldsymbol{K}^{N}$.

Claim 4.7. If I is brimful as a linear order, $E M_{\tau}(I, \Phi)$ is brimful as a member of K.

Proof. Let $M=E M(I, \Phi)$; we must show $M \uparrow \tau$ is brimful as a member of $\boldsymbol{K}$. Suppose $M_{1} \preceq \boldsymbol{K} M \uparrow \tau$ with $\left|M_{1}\right|=\sigma<|M|$. Then there is $N_{1}=E M\left(I^{\prime}, \Phi\right)$ with $\left|I^{\prime}\right|=\sigma$ and $M_{1} \subseteq N_{1} \leq M$. By Lemma 2.7.5, $N_{1} \uparrow \tau \preceq \boldsymbol{K} M \uparrow \tau$. So $M_{1} \preceq \boldsymbol{K} N_{1} \uparrow$ $\tau$ by the coherence axiom. Let $M_{2}$ have cardinality $\sigma$ and $M_{1} \preceq \boldsymbol{K} M_{2} \preceq \boldsymbol{K} M \uparrow \tau$. Choose a $\tau^{\prime}$-substructure $N_{2}$ of $M$ with cardinality $\sigma$ containing $N_{1}$ and $M_{2}$. Now, $N_{2}$ can be embedded by a map $f$ into the $\sigma$-universal $\tau^{\prime}$-structure $N_{3}$ containing $N_{1}$ which is guaranteed by Claim 4.6. But $f\left(N_{2}\right) \uparrow \tau \preceq K_{3} \uparrow \tau$ by the coherence axiom so $N_{3}\left\lceil\tau\right.$ is the required $\sigma$-universal extension of $M_{1} . \quad \square_{4.7}$

Definition 4.8. (1) Let $N \subset \mathbb{M} . N$ is $\lambda$-Galois-stable if for every $M \subset N$ with cardinality $\lambda$, only $\lambda$ Galois types over $M$ are realized in $N$.

(2) $\boldsymbol{K}$ is $\lambda$-Galois-stable if $\mathbb{M}$ is. That is aut . $_{M}(\mathbb{M})$ has only $\lambda$ orbits for every $M \subset \mathbb{M}$ with cardinality $\lambda$.

Since we are usually working in an AEC, we will frequently abuse notation and write stable rather than Galois-stable.

Since for brimful $I$, a $M=E M(I, \phi)$ is brimful, and for $M_{0} \preceq \boldsymbol{K} M_{1} \preceq \boldsymbol{K} M$, each Galois type over $M_{0}$ realized in $M$ is represented by an $M_{1}$ with $\left|M_{1}\right|=\left|M_{0}\right|$, Claim 4.7 implies immediately:

Claim 4.9. If $\boldsymbol{K}$ is $\lambda$-categorical, the model $M$ with $|M|=\lambda$ is $\sigma$-Galois stable for every $\sigma<\lambda$.

THEOREM 4.10. If $\boldsymbol{K}$ is categorical in $\lambda$, then $\boldsymbol{K}$ is $\sigma$-Galois-stable for every $\sigma<\lambda$.

Proof. Suppose $\boldsymbol{K}$ is not $\sigma$-stable for some $\sigma<\lambda$. Then by Löwenheim-Skolem, there is a model $N$ of cardinality $\sigma^{+}$which is not $\sigma$-stable. Let $M$ be the $\sigma$ stable model with cardinality $\lambda$ constructed in Claim 4.9. Categoricity and joint embedding imply $N$ can be embedded in $M$. The resulting contradiction proves the result. $\square_{4.10}$

Corollary 4.11. Suppose $\boldsymbol{K}$ is categorical in $\lambda$ and $\lambda$ is regular. The model of power $\lambda$ is saturated and so model homogeneous.

Proof. Choose in $M_{i} \preceq \boldsymbol{K} \mathbb{M}$ using $<\lambda$-stability and Löwenheim-Skolem, for $i<\lambda$ so that each $M_{i}$ has cardinality $<\lambda$ and $M_{i+1}$ realizes all types over $M_{i}$. By regularity, it is easy to check that $M_{\lambda}$ is saturated. $\quad \square_{4.11}$ 
The same argument gives saturated models in smaller regular cardinals; more strongly we can demand that the saturated model be an Ehrenfreuht-Mostowski model.

Corollary 4.12. Suppose $\boldsymbol{K}$ is an AEC with vocabulary $\tau$ that is categorical in $\lambda$ and $\lambda$ is regular. Then for every regular $\mu, \operatorname{LS}(\boldsymbol{K})<\mu<\lambda$ there is a model $M_{\mu}=E M_{\tau}\left(I_{\mu}, \Phi\right)$ which is saturated. In particular, it is $\mu$-model homogeneous.

Proof. For any ordered set $J$ of cardinality $\lambda$, let $M=E M_{\tau}(J, \phi)$ be the model of cardinality $\lambda$. We construct an alternating chain of $\boldsymbol{K}$-submodels of length

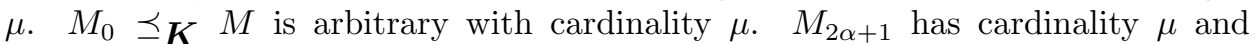
realizes all types over $M_{2 \alpha}$ (possible by Corollary 4.10. $M_{2 \alpha+2}$ has cardinality $\mu, M_{2 \alpha+1} \preceq \boldsymbol{K} M_{2 \alpha+2}$ and $M_{2 \alpha+2}$ is $E M_{\tau}\left(I_{\alpha+1}, \Phi\right)$ where $I_{\alpha} \subset I_{\alpha+1} \subset J$ and all $I_{\alpha}$ have cardinality $\mu$. Then $E M_{\tau}\left(I_{\mu}, \Phi\right)=\bigcup_{\alpha<\mu} E M_{\tau}\left(I_{\alpha}, \Phi\right)$ is saturated by regularity. $\square_{4.12}$

Now using stability we can get a still stronger result, eliminating the hypothesis that $\mu$ is regular. We show the proofs of both Corollary 4.12 and Corollary 4.13 since in the first case we constructed a saturated model directly and in the second a model homogeneous structure.

Corollary 4.13. Suppose $\boldsymbol{K}$ is an AEC with vocabulary $\tau$ that is categorical in $\lambda$ and $\lambda$ is regular. Then for every $\mu, \operatorname{LS}(\boldsymbol{K})<\mu<\lambda$ there is a model $M_{\mu}=$ $E M_{\tau}\left(\mu^{<\omega}, \Phi\right)$ which is $\mu$-model homogeneous.

Proof. Represent the categoricity model as $M^{*}=E M_{\tau}\left(\lambda^{<\omega}, \Phi\right)$. We show $M_{\mu}=$ $E M_{\tau}\left(\mu^{<\omega}, \Phi\right)$ is model homogenous. Suppose $M_{1} \preceq \boldsymbol{K} M_{\mu}$ with $\left|M_{1}\right|=\sigma<\left|M_{\mu}\right|$. Then there is $N_{1}=E_{\tau}\left(I_{1}, \Phi\right)$ with $\left|I_{1}\right|=\sigma, M_{1} \subset N_{1}$ and $I_{1} \subset \mu^{<\omega}$. Let $M_{2}$ have cardinality $\sigma$ and $M_{1} \preceq \boldsymbol{K} M_{2}$. By amalgamation, choose $N_{2} \in \boldsymbol{K}$ which is an amalgam of $N_{1}$ and $M_{2}$ over $M_{1}$. By the $\lambda$-model homogeneity of $M^{*}$, there is an embedding of $N_{2}$ into $M^{*}$ over $N_{1}$ say with image $N_{2}^{\prime}$. Then $N_{2}^{\prime} \subset E M_{\tau}(J, \Phi)$ for some $J$ with $I_{1} \subset J \subset \lambda^{<\omega}$ and $|J|=\sigma$. Now by Corollary 4.5 and an argument like that in Claim 4.7, there is an embedding of $E M_{\tau}(J, \Phi)$ into $M=E M_{\tau}\left(\mu^{<\omega}, \Phi\right)$ over $N_{1}$, and a fortiori over $M_{1}$ and we finish. $\square_{4.13}$

REMARK 4.14. (1) Note that for each $\sigma$ less than the categoricity cardinal $\lambda$, the $\sigma$-universal model that is constructed has the form $E M_{\tau}\left(I^{\prime}, \Phi\right)$ for some $I^{\prime}$.

(2) Compare Claim 4.13 to I.3.1 in [18], which has the same conclusion but weakening the amalgamation propery to: there are no maximal models. There are two uses of the amalgamation property in the argument for Claim 4.13. The first requires only that $M_{1}$ be an amalgamation base for models in $\boldsymbol{K}$ of size $\mu$ and so extends easily to prove the analogous result where $\boldsymbol{K}$ has amalgamation is replaced by $\boldsymbol{K}$ has no maximal models. The second is that $M$ is $<\lambda$ model homogenous. This step is done in quite a different way in the proof of I.3.1 in [18]; stability is not used but GCH is. 


\section{Morley's method for Galois Types}

Now we prove 'Morley's method' for Galois types.

Lemma 5.1. [II.1.5 of Sh394] If $M_{0} \leq M$ and $M$ is substantial with respect to $\left|M_{0}\right|$, we can find an EM-set $\Phi$ such that the following hold.

(1) The $\tau$-reduct of the Skolem closure of the empty set is $M_{0}$.

(2) For every $I, M_{0} \leq E M(I, \Phi)$.

(3) If $I$ is finite, $E M_{\tau}(I, \Phi)$ can be embedded in $M$.

(4) $E M_{\tau}(I, \Phi)$ omits every galois type over $M_{0}$ which is omitted in $M$.

Proof. Let $\tau_{1}$ be the Skolem language given by the presentation theorem and consider $M$ as the reduct of $\tau_{1}$ structure $M^{1}$. Add constants for $M_{0}$ to form $\tau_{1}^{\prime}$. Now apply Lemma 2.6 to find an EM-diagram $\Phi$ (in $\tau_{1}^{\prime}$ ) with all $\tau$-types of finite subsets of the indiscernible sequence realized in $M$. Now 1) and 2) are immediate. 3 ) is easy (using clause 5 of Theorem 2.7) since we chose $\Phi$ so all finite subsets of the indiscernible set (and so their Skolem closures) are realized in $M$.

The omission of Galois types is more tricky. Consider both $M$ and $N=E M_{\tau}(I, \Phi)$ embedded in $\mathbb{M}$. Let $N^{1}$ denote the $\tau_{1}^{\prime}$-structure $E M(I, \Phi)$. We need to show that if $a \in N, p=\mathrm{ga}-\operatorname{tp}\left(\mathrm{a} / \mathrm{M}_{0}\right)$ is realized in $M$. For some $\mathbf{e} \in I, a$ is in the $\tau_{1}$-Skolem hull $N_{\mathbf{e}}$ of e. (Recall the notation from the presentation theorem.) By 3 ) there is an embedding $\alpha$ of $N_{\mathbf{e}}$ into $M^{1}$ over $M_{0} . \alpha$ is also an isomorphism of $N_{\mathbf{e}} \uparrow \tau$ into $M$. Now, by the model homogeneity, $\alpha$ extends to an automorphism of $\mathbb{M}$ fixing $M_{0}$ and $\alpha(a) \in M$ realizes $p . \quad \square_{5.1}$

This has immediate applications in the direction of transferring categoricity.

TheOrem 5.2. Suppose $M \in \boldsymbol{K}$ omits a Galois type $p$ over a submodel $M_{0}$ with $|M| \geq \mu\left(\left|M_{0}\right|\right)$. Then there is no regular cardinal $\lambda \geq|M|$ in which $\boldsymbol{K}$ is categorical.

Proof. By Lemma 5.1, there is a model $N \in \boldsymbol{K}$ with cardinality $\lambda$ which omits $p$. But by Lemma 4.11, the unique model of power $\lambda$ is saturated. $\quad \square_{5.2}$

\section{Tameness and Downwards Categoricity}

In [12] Shelah asserts the following result:

THEOREM 6.1. If $\boldsymbol{K}$ is categorical in a regular cardinal $\lambda$ and $\lambda>\mu(\mu(|\tau|))$ then $\boldsymbol{K}$ is categorical in every $\theta$ with $\mu(\mu(|\tau|)) \leq \theta \leq \lambda$.

Here is a sketch of the argument. We have shown that there are saturated models of power $\theta$ for every $\theta<\lambda$. The obstacle to deducing downward categoricity is that Theorem 5.1 only allows us to transfer the omission of types when the model omitting the type is much bigger than the domain of the type. The first step in remedying this problem is to show that all types are determined by 'relatively small' subtypes. More precisely, we need the notion that Grossberg and Van Dieren [6] have called $\chi$-tame and Shelah $[\mathbf{1 2}]$ refers to has 'having $\chi$-character'. We add an extra parameter to be careful. 
Definition 6.2. We say $\boldsymbol{K}$ is $(\chi, \mu)$-tame if for any saturated $N \in \boldsymbol{K}$ with $|N|=$ $\mu<\lambda$ if $p, q, \in \mathrm{ga}-\mathrm{S}(\mathrm{N})$ and for every $N_{0} \leq N$ with $\left|N_{0}\right| \leq \chi, p\left\lceil N_{0}=q\left\lceil N_{0}\right.\right.$ then $q=p$.

Shelah asserts the following in Sections II.1 and II.2.3 of the published version of [12]. The published proof is incomplete; I haven't yet seen the corrections. But it seems to use only Ehrenfeucht-Mostowski type methods.

Theorem 6.3. Suppose $\boldsymbol{K}$ is $\lambda$-categorical for $\lambda \geq \mu(\tau)$ and $\lambda$ is regular. Then $\boldsymbol{K}$ is $\left(\chi, \chi_{1}\right)$-tame for some $\chi$ and any $\chi_{1}$ with $\chi<\mu(\tau) \leq \chi_{1} \leq \lambda$.

The naive argument would give $\chi=\mu(\tau)$ since one is omitting types. But omitting in every cardinal below $\mu(\tau)$ is as good as in $\mu(\tau)$ so the conclusion becomes for some $\chi$ with $\chi<\mu(\tau)$.

The remainder of the argument for Theorem 6.1 uses such technologies as splitting and minimal types that are beyond the scope of this paper.

Since we were expounding $[\mathbf{1 2}]$ we assumed, as there, that $\boldsymbol{K}$ has arbitrarily large models and the amalgamation and joint embedding properties. We used amalgamation heavily to get monster models and thus get the group theoretic definition of Galois-type. By using the more complicated definition of a Galois type as an equivalence relation on triples, many of these notions can be extended to classes without amalgamation. And one can even prove $[\mathbf{1 5}, \mathbf{1}]$, saturation equals model homogeneity with no amalgamation hypothesis whatsoever. However, I don't know anyway to prove the existence of either saturated model homogeneous models in general AEC without at least some amalgamation hypothesis.

\section{References}

[1] J.T. Baldwin. Notes on aec. Available at www.math.uic.edu/ jbaldwin, 200?

[2] Steven Buechler. Essential Stability Theory. Springer-Verlag, 1991.

[3] C. C. Chang. Some remarks on the model theory of infinitary languages. In J. Barwise, editor, The syntax and semantics of infinitary languages, pages 36-64. Springer-Verlag, 1968. LNM 72 .

[4] C.C. Chang and H.J Keisler. Model theory. North-Holland, 1973.

[5] R. Grossberg. A course in model theory. preprint.

[6] R. Grossberg and M. Van Dieren. Galois stability for tame abstract elementary classes. preprint.

[7] W. Hodges. Model Theory. Cambridge University Press, 1993.

[8] H.J Keisler. Model theory for Infinitary Logic. North-Holland, 1971.

[9] J. A. Makowsky. Abstract embedding relations. In J. Barwise and S. Feferman, editors, ModelTheoretic Logics, pages 747-792. Springer-Verlag, 1985.

[10] M. Morley and R.L. Vaught. Homogeneous universal models. Mathematica Scandinavica, 11:37-57, 1962.

[11] S. Shelah. Classification Theory and the Number of Nonisomorphic Models. North-Holland, 1978.

[12] S. Shelah. Categoricity for abstract classes with amalgamation. Annals of Pure and Applied Logic, 98:261-294, 1999. paper 394.

[13] S. Shelah. Categoricity of abstract elementary classes: going up inductive step. preprint 600, 200 ?

[14] S. Shelah. On categorical a.e.c. preprint 734, 200?

[15] S. Shelah. Categoricity of abstract elementary class in two successive cardinals. Israel Journal of Mathematics, 126:29-128, 2001. paper 576. 
1HOHN T. BALDWIN DEPARTMENT OF MATHEMATICS, STATISTICS AND COMPUTER SCIENCE UNIVERSITY OF ILLINOIS AT C

[16] S. Shelah and O. Kolman. Categoricity of theories in $L_{\kappa, \omega}$, when $\kappa$ is a measurable cardinal. part 1. preprint 362 .

[17] Saharon Shelah. Classification of nonelementary classes II, abstract elementary classes. In J.T. Baldwin, editor, Classification theory (Chicago, IL, 1985), pages 419-497. Springer, Berlin, 1987. paper 88: Proceedings of the USA-Israel Conference on Classification Theory, Chicago, December 1985; volume 1292 of Lecture Notes in Mathematics.

[18] Saharon Shelah and Andrés Villaveces. Toward categoricity for classes with no maximal models. Annals of Pure and Applied Logic, 97:1-25, 1999. revised version : www. math. rutgers. edu/ ${ }^{\text {shelah. }}$

[19] Monica VanDieren. Categoricity and stability in abstract elementary classes. PhD thesis, Carnegie Mellon University, 2002. 